\title{
Design Optimization of Reflectarray Antenna Fabricated above Paper Based Substrate Materials
}

\author{
Hasan Ijaz Malik ${ }^{1}$, Muhammad Yusof Ismail ${ }^{1, *}$ \& Muhammad Hezri Mokhtar ${ }^{2}$ \\ ${ }^{1}$ Faculty of Electrical and Electronic Engineering, ${ }^{2}$ Faculty of Engineering Technology \\ Universiti Tun Hussein Onn Malaysia (UTHM), Parit Raja, 86400, Batu Pahat, Johor MALAYSIA \\ *Corresponding Author
}

DOI: https://doi.org/10.30880/ijie.2019.11.06.016

Received 7 May 2018; Accepted 26 November 2018; Available online 12 September 2019

\begin{abstract}
A novel combination of X - band reflectarray antenna printed above paper based substrate material has been presented for microwave antenna application. Paper substrate materials with controlled compositions, characterized using dielectric probe shows dielectric constants of 1.68 and 1.74 and loss tangents of 0.074 and 0.082 respectively. Reflectarray unit elements simulated using CST MWS were fabricated over the paper substrates using adhesive copper tape. The fabricated samples tested for the scattering parameters demonstrate an efficient bandwidth of 340 and $290 \mathrm{MHz}$ for both the substrate materials. Moreover the measured reflection phase of the elements covers a phase range of $310^{\circ}$ and $290^{\circ}$ for proposed paper substrates.
\end{abstract}

Keywords: Reflectarray antenna, Paper substrate, Broadband, Figure of Merit

\section{Introduction}

Microstrip reflectarray antennas present themselves as a low profile, cost efficient solution for high gain antenna applications. They combine the key features of a parabolic dish reflector and the planar phased array antennas. The primary application of these high gain directive antennas is the radar antennas and long range directional beams. A microstrip reflectarray antenna consists of an array of printed microstrip patches over a suitable dielectric substrate material. The array is fed spatially by a feed horn. Each element on the array is designed individually, according to its spatial position with respect to feeding antenna. Thus converging the incident energy of feed in a concentrated beam at a far field region.

Besides the low profile and manufacturing ease of microstrip reflectarray antenna, one of the major limitations of the of microstrip antenna technology is intrinsic narrow bandwidth. In the literature different techniques have been proposed for bandwidth improvement of printed reflectarray antenna technology. Stacked element configurations with two and three layers stacked layer of elements have been proposed with variable size element [1]-[3]. However the stacked configuration of elements is generally suited for fixed beam applications and it requires precise alignment and assembling of microstrip layers for fruitful results. Reflectarray element with aperture coupled delay lines have also been proposed for bandwidth improvement in X-band. The delay lines attached to ring cut patches control the phases of the individual elements [4]. However the microstrip lines attached to the ring patch may result in spurious radiation and contribute in reduction of antenna efficiency.

Different types of dual band and dual frequency element configuration have also been suggested for bandwidth improvement and multi-band operation [5]-[7]. In that work dual resonance behaviour has been proposed using split ring slots [5], parallel dipole element configuration [6] and a slotted circular patch with delay lines attached [7]. The dual resonance operation requires two feeding configurations for the reflector and makes the design process complex. 
In order to address the narrow bandwidth behaviour of microstrip reflectarray, this article presents a novel combination of microstrip reflectarray constructed on cellulose based paper substrate. The organic paper based substrate material offers a low dielectric constant that will help improve the radiation characteristics of reflectarray element. Paper substrate materials have been manufactured under controlled composition of recycled materials to acquire desired permittivity values. A brief overview of different types of losses associated with microstrip reflectarray antenna is presented. The proposed material was characterized using a broadband dielectric assessment technique. Surface current density and the electric field intensity of reflectarray elements on proposed paper substrate has been analysed using CST MWS software platform. Rectangular patch elements with X-band frequency operations were simulated and fabricated over the proposed paper based substrate material. Reflectarray unit elements were tested using a waveguide simulated technique taking into consideration of an infinite array of elements [8].

\section{Structure}

In a microstrip reflectarray antenna the loss in the complete array is a combination of more than one factor. The total incident energy over the array from the feed horn can be represented as a combination of reflected energy and the energy lost in the reflectarray antenna:

$$
\bar{E}_{\text {lne }}=\bar{E}_{\text {ref }}+\bar{E}_{\text {loss }}
$$

Where $\bar{E}_{\text {logs }}$ represents the losses associated with the reflectarray antenna. The prominent losses associated with the reflectarray can be differentiated as:

$$
\bar{E}_{\text {loss }}=\bar{E}_{\text {asse }}+\bar{E}_{\text {mat }}
$$

Where $\bar{E}_{\text {asse }}$ is the energy lost in the losses related to the structrure of the reflectarray antenna, such as spill over loss, illumination loss and the losses related to feed antenna. These losses can be minimized by proper feed placement and assembly alignments. $\bar{E}_{\text {mat }}$ represents the energy lost due to the material properties of reflectarray antenna and in case of a conductive patch backed by a grounded dielectric substrate, it can be stated as:

$$
\bar{E}_{\text {mat }}=\alpha_{c}+\alpha_{d}
$$

The $\bar{E}_{\text {mat }}$ can be further divided in to conductor loss $\alpha_{e}$ and the dielectric loss $\alpha_{d}$. The conductor loss is due to finite conductivity of the conductive material and it can be found by:

$$
\alpha_{c}=\frac{8.86}{W Z_{m}} \sqrt{\frac{\omega \mu_{0}}{2 \sigma_{c}}}
$$

Where $Z_{m}$ is the patch impedance, $\omega$ is the operational frequency and $\sigma_{c}$ is the bulk conductivity of the material. It can be seen that the conductor loss depends upon the operational frequency and it increases with the increase in frequency. The reason of increased conductor loss is the exponential reduction of skin depth for the moving charge carriers in the conductor as the frequency increases. The other part of material losses in microstrip patch antenna is the dielectric loss and it is stated as:

Where

$$
\alpha_{d}=\frac{\omega}{2} \sqrt{\mu_{0} \varepsilon_{0} \varepsilon_{y}} \tan \delta
$$

$$
\tan \delta=\frac{\varepsilon_{\gamma}}{\varepsilon^{v}}
$$

Equation (5) shows that the dielectric loss depends upon the loss tangent as well as the dielectric material permittivity. The term $\tan \delta$ is the ratio of imaginary and real parts of permittivity and it can be interpreted as the phase delay that rises between the excitation and the response of dipoles in the dielectric region as a time varying signal is applied to them. The more the phase delay the more is the resistivity of material to switch poles with respect to external applied rises between the excitation and the response of dipoles in the dielectric region as a time varying signal is applied to them. The more the phase delay the more is the resistivity of material to switch poles with respect to external applied field. 
In the case of microstrip reflectarray dielectric loss $\alpha_{d}$ is due to the multiple bounces of the $\mathrm{E}$ - field in the region between the patch and the ground plane. The loss reduces with the increase in the substrate height, caused by reduction of multiple bounces in the dielectric region. The loss in the substrate region can also be explained in term of cavity model approach. A dielectric slab between a ground plane and patch element is analogous to a dielectric loaded waveguide assuming no fringing fields exist at the sides. Since in a waveguide besides the dominant mode of operation, there are other parasitic modes also that result in the loss of power. As the permittivity of loaded dielectric material is increased the number of higher order modes increases and the loss is increased due to parasitic effect. Thus a low permittivity dielectric material is desired to reduce the losses in the substrate region due to dielectric loss and also to enhance the fringing effect of the patch element.

\section{Material Characterization}

The proposed cellulose based dielectric material was first characterized using a Speag dielectric assessment kit. The kit consists of a dielectric probe, for broadband analysis of material properties analysis. The DAK 3.5 probe was used for material assessment and it offers an analysis over a frequency range of 0.2 to $20 \mathrm{GHz}$. The set-up uses an external software platform installed over a computer to handle the probe and the vector network analyser. The material properties measurement set-up of the dielectric is shown in Fig. 1. Fig 1(a) shows the proposed sample of two paper based substrate materials (sample 1: S1 and sample 2: S2) with other commercially available substrates. Multiple layers of substrate were glued together to achieve a suitable substrate thickness.

The samples were passed through a thorough heating and drying process to eradicate the moisture content in the paper substrate. Fig. 1(b) shows the set-up of the material characterization where a dielectric probe is attached to a vector network analyser, the VNA was connected to the software platform running on laptop. The probe parameters, analysis range and calibration feature were handled by the software platform. The dielectric probe used air, copper strip and water for open, short and load calibrations respectively. Fig. 1(c) shows the material under test All figures should be numbered with Arabic numerals $(1,2,3 \ldots)$. Every figure should have a caption. All photographs, schemas, graphs and diagrams are to be referred to as figures. Line drawings should be good quality scans or true electronic output. Lowquality scans are not acceptable. Figures must be embedded into the text and not supplied separately. In MS word input the figures must be properly coded. Preferred format of figures are PNG, JPEG, GIF etc. Lettering and symbols should be clearly defined either in the caption or in a legend provided as part of the figure. Figures should be placed at the top or bottom of a page wherever possible, as close as possible to the first reference to them in the paper. Please ensure that all the figures are of 300 DPI resolutions as this will facilitate good output.

The figure number and caption should be typed below the illustration in $8 \mathrm{pt}$ and left justified [Note: one-line captions of length less than column width (or full typesetting width or oblong) centered]. For more guidelines and information to help you submit high quality artwork please visit: http://www.elsevier.com/artworkinstructions Artwork has no text along the side of it in the main body of the text. However, if two images fit next to each other, these may be placed next to each other to save space. For example, see Fig. 1.
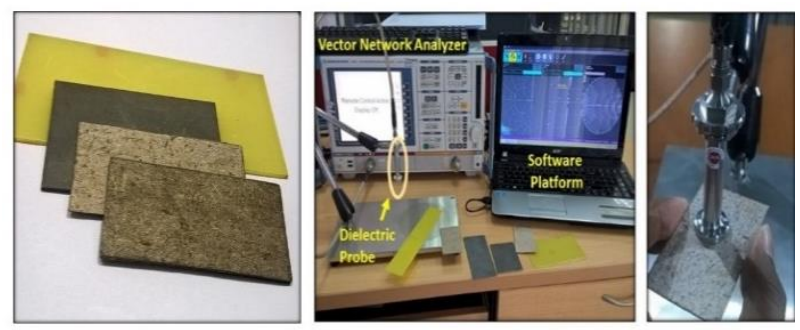

Fig. 1 - Dielectric material characterization (a) Proposed paper substrates (b) Set-up of dielectric assessment kit (c) Material under test for assessment. 


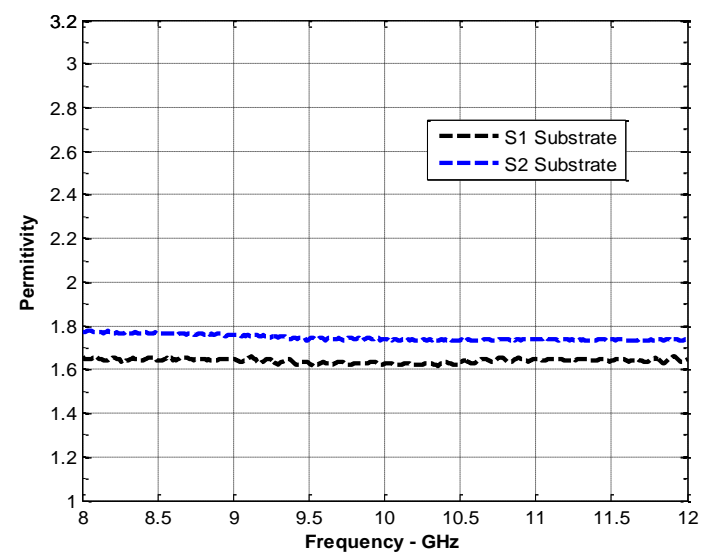

(a)

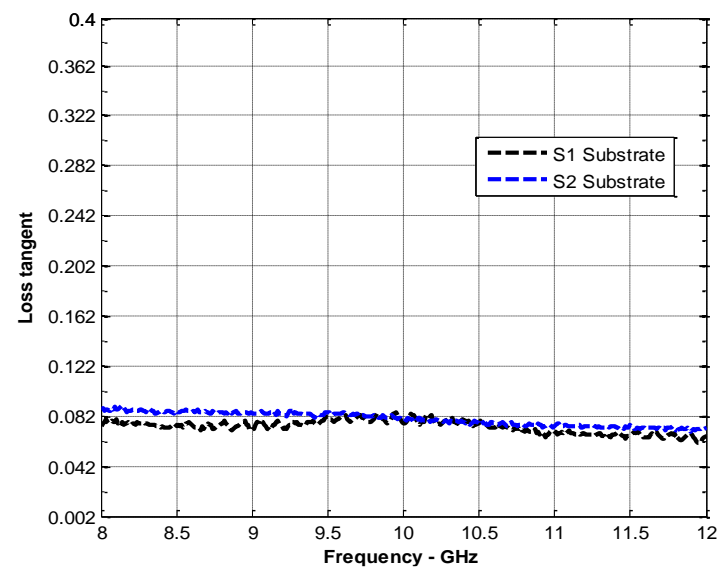

(b)

Fig. 2 - Dielectric material characterization (a) Dielectric constant (b) Loss tangent.

The sample under consideration was pressed with the face of dielectric probe to achieve a stable measurement of dielectric constant and loss tangent. Multiple measurements were performed and a mean value was selected. The results of dielectric material characterization are presented in Fig. 2. The dielectric permittivity results for the proposed substrate material are shown in Fig. 2(a). Fig. 2(b) shows the loss tangent results deduced from the material characterization. Table I summarizes the results of the material characterization for paper substrate samples.

Table 1 - Paper substrate material characterization.

\begin{tabular}{cccc}
\hline Material & $\varepsilon_{\mathrm{r}}$ & $\tan \delta$ & $\begin{array}{c}\text { Thickness } \\
(\mathrm{mm})\end{array}$ \\
\hline S1 & 1.68 & 0.074 & 1.40 \\
\hline S2 & 1.74 & 0.082 & 2.30 \\
\hline
\end{tabular}

As shown in Table 1, S1 and S2 substrate materials show dielectric permittivities of 1.68 and 1.74 respectively. Moreover S1 and S2 substrate material depict loss tangents values of 0.074 and 0.082 respectively. The samples used for the dielectric material characterization were 1.40 and $2.30 \mathrm{~mm}$ thick for S1 and S2 substrates respectively.

\section{Electrical Field and Surface Current Distribution}

After identification of the material properties of the paper substrate materials unit reflectarray elements were simulated for analysis using commercially available CST MWS software. Rectangular patch elements with periodic boundary conditions were simulated for realizing an infinite array of reflectarray elements. The dominant mode for mode of excitation was selected as $\mathrm{TE}_{10}$ with vertical excitation of E-fields in X-band frequency range. Surface current distribution and electric field intensity of patch element were simulated and analysed for different substrate thickness to monitor the effect of substrate height. 
Fig. 3 shows the plot presenting the effect of variable substrate height on the surface current density. The curves show a decreasing trend of surface current density with increase in the substrate height. The surface current density for $\mathrm{S} 1$ substrate decreases from 262 to $102.9 \mathrm{~A} / \mathrm{m}$ as the substrate height is swept from 0.2 to $3 \mathrm{~mm}$. Both the substrate materials show similar trends as shown in Fig. 3, for S2 substrate the surface current decreased from 230 to 97.4 A/m as the substrate thickness is increased from 0.2 to $3.0 \mathrm{~mm}$. The electric field intensity of the patch element was also analysed and compared with variable substrate heights. The E-field intensity inside the cavity between the patch element and the ground plane decreases with the increase in substrate height.

Fig. 4 shows the effect of changing substrate height over the electric field intensity. A decreasing trend of electric field concentration can be seen with the increased substrate height. The E-field for S1 substrate gradually decreases from 28.3 to $4.3 \mathrm{KV} / \mathrm{m}$ as the substrate height increases from 0.2 to $3.0 \mathrm{~mm}$. Similarly, for $\mathrm{S} 2$ substrate varying the substrate height from 0.2 to $3 \mathrm{~mm}$. The decrease in the surface current density and the electric field intensity can be explained by the relationship between surface current density and the electric field intensity as:

$$
\vec{l}=\sigma \vec{E}
$$

Where $\vec{f}$ is the surface current density, $\sigma$ is the conductivity of the material and $\vec{E}$ is the electric field intensity between the patch and the ground plane. Thus by the increase in the substrate height the density of the electric field in the region decreases and the surface current density also decreases proportionally keeping the conductivity constant. The simulated samples with rectangular patch element show a maximum loss at the resonance point [9]. The loss is maximum at the resonance, since at the resonance electric field intensity is shown to be highest in the region in between the ground and patch element. The high surface current distribution of charges on the patch element contributes to the conductor losses in the patch. The conductor loss is also maximum at the resonance since at the resonance majority of the charge carriers are present. Due to these high losses at the resonant frequency point of the patch the reflection loss is also maximum at the resonance. The loss can be reduced by increasing the substrate height on the patch, however increasing the substrate height will generate the lossy surface waves. So a trade-off is to be made for the substrate height and the losses.

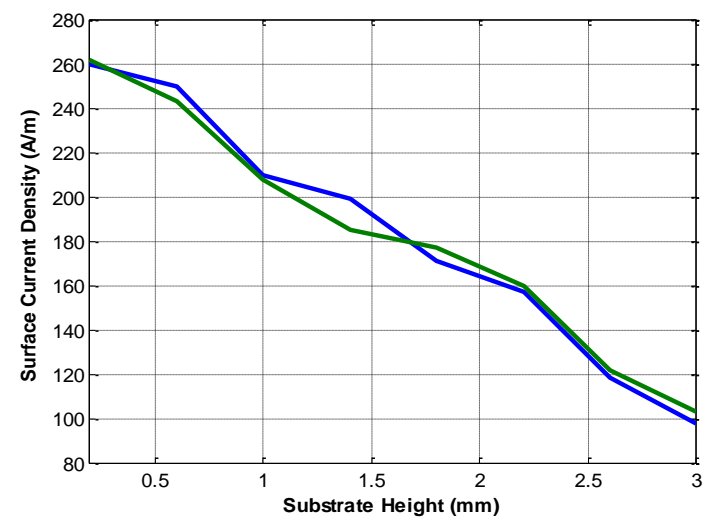

Fig. 3 - Effect of variable substrate height on surface current density.

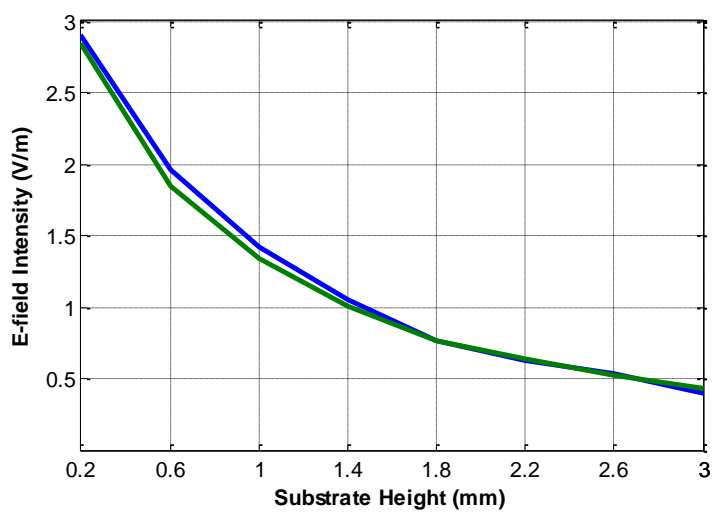

Fig. 4 - Effect of variable substrate height on the E-field intensity of patch element. 


\section{Fabrication and Measurement}

In order to validate the paper substrate material reflectarray antennas, unit cells of rectangular patch elements were fabricated on both S1 and S2 substrate materials. In order to obtain better conductivity performance a $70 \mu \mathrm{m}$ adhesive thick copper tape was used for the fabrication of elements. The copper tape offers the conductivity of bulk copper i.e. $5.8 \times 10^{7} \mathrm{~S} / \mathrm{m}$ compared to silver nano-particles ink [10]. The elements were tested for the scattering parameters using a tapered open ended X-band waveguide. The perfect electric and magnetic boundaries of the waveguide, realizing the effect of an infinite array of reflectarray element. Multiple samples were fabricated to ensure repeatability of the results. Fig. 5 shows the fabricated samples and the measurement set-up. Fig. 5(a) and 5(b) show the fabricated rectangular patch elements on S1 and S2 substrates respectively. Fig. 5(c) shows the aperture of the waveguide simulator where the elements are placed for measurements. The typical X-band Agilent adopter attached to the tapered waveguide has been shown in Fig. 5(d). The complete measurement set-up of the reflectarray has been shown in Fig. 5(e) where the waveguide is attached to vector network analyzer and the elements are placed in the waveguide aperture for scattering parameter measurements.

A comparison between measured and simulated results is presented for the validation of proposed paper substrate material. Scattering Parameters were measured for each of the reflectarray elements. Fig. 6 shows the measured reflection loss results comparison with the simulated results from simulated CST MWS model. The reflection loss comparisons of S1 and S2 substrate materials has been shown in Fig. 6, where the maximum reflection loss is shown at the resonance point. The fabricated rectangular patch elements on S1 and S2 substrate materials resonate at 9.51 and $9.68 \mathrm{GHz}$ respectively.

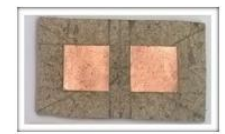

(a)

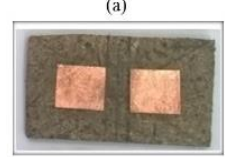

(b)

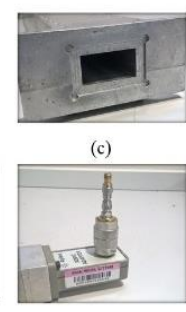

(d)

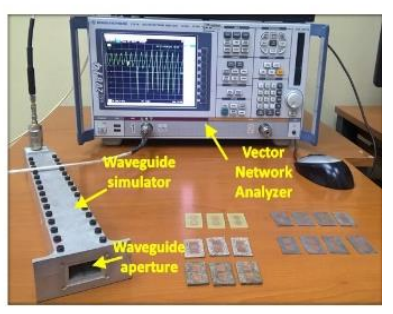

(e)

Fig. 5 - Measurement set-up (a) S2 sample (b) S1 sample (c) Aperture of X-band waveguide simulator (d) An Xband adapter for waveguide (e) Complete measurement set-up with VNA attached to waveguide simulator.

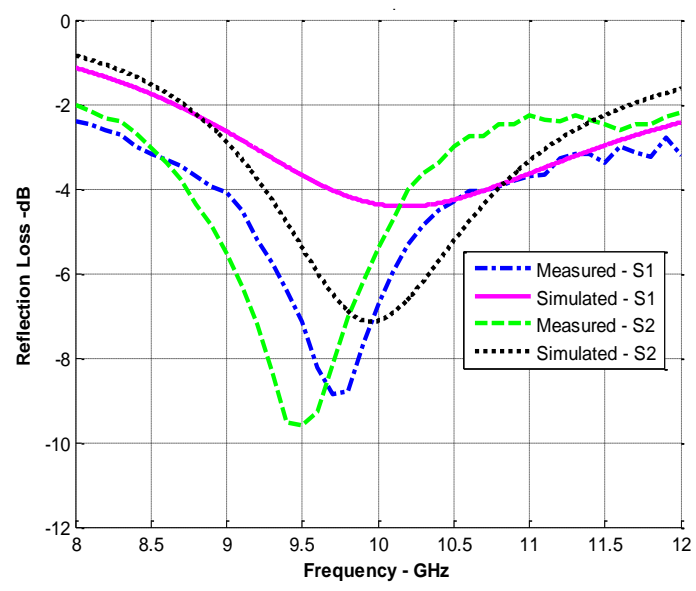

Fig. 6 - Comparison between reflection loss curves for simulated and measured results.

Fig. 7 presents the reflection phase comparison of proposed elements designs on S1 and S2 substrate materials. The comparison between measured and simulated results for scattering parameters of S1 and S2 substrate materials is presented in Table 2 . 


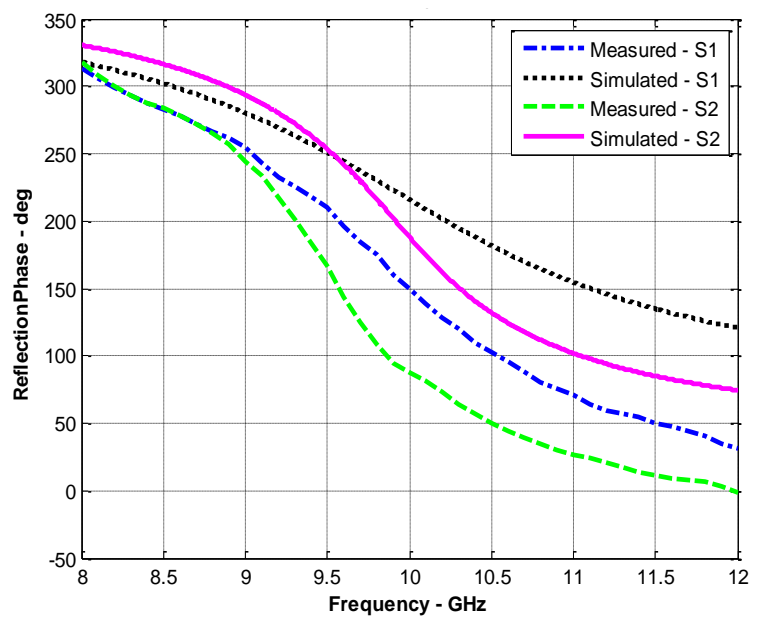

Fig. 7 - Comparison between measured and simulated reflection phase results.

Table 2 - Comparison between measured and simulated results for paper substrate materials.

\begin{tabular}{|c|c|c|c|c|}
\hline Material & $\begin{array}{c}f_{r} \\
\mathrm{GHz}\end{array}$ & $\begin{array}{l}\text { RL } \\
\mathrm{dB}\end{array}$ & $\begin{array}{c}\Delta f \\
\mathrm{MHz}\end{array}$ & $\begin{array}{c}\Delta \Phi \\
\text { Deg }\end{array}$ \\
\hline \multicolumn{5}{|l|}{ S1 } \\
\hline Simulated & 9.96 & -7.17 & 580 & 275 \\
\hline Measured & 9.51 & -9.60 & 340 & 310 \\
\hline \multicolumn{5}{|l|}{ S2 } \\
\hline Simulated & 10.18 & -4.41 & 680 & 220 \\
\hline Measured & 9.68 & -8.85 & 290 & 294 \\
\hline
\end{tabular}

Comparison between measured and simulated results show that the substrates S1 and S2 present 340 and $290 \mathrm{MHz}$ measured $10 \%$ bandwidth respectively. Moreover the measured phase range for both the substrate materials is $310^{\circ}$ and $294^{\circ}$ respectively. The measured phase range is greater than simulated range due to increase in loss for measured results. There is a $5 \%$ frequency shift between measured and simulated resonance. This deviation in the measured results might be due to dimensional errors caused by fabrication tolerances.

A comparison of proposed paper substrate with commercially available substrate materials such as Rogers Duroid 5880 and FR-4 epoxy substrate presents an intermediate performance. The proposed substrate material present 1.68 and 1.74 dielectric permittivities compared to 2.2 and 4.7 presented by Rogers Duroid and FR-4 material respectively. However the proposed substrates present a higher loss tangent value compared to Rogers Duroid, which has a dielectric loss tangent of 0.001. Both the presented materials show excellent bandwidth improvements compared to conventional materials used for reflectarrays. Thus, a trade-off is to be adopted between the cost and efficiency for the selection of a suitable substrate material. For a long term operation of propose substrate suitable laminations must be applied to the reflectarrays to avoid performance deterioration of the substrate material by environment hostilities. Thin polythene laminations does not affect the radiation characteristics of paper substrate antennas [11].

\section{Conclusion}

The paper presents a wideband solution to intrinsic narrow bandwidth limitation of microstrip reflectarray antenna. Organic substrate material has been introduced for the first time for reflectarray antenna. Organic paper based material made up of recycled material, has been characterized using dielectric probe method. The substrate materials show dielectric constant of 1.68 and 1.74 with a loss tangent of 0.074 and 0.082 . Simulation of rectangular patch elements was carried out using CST MWS. Testing of fabricated samples was done using a waveguide simulator technique. The proposed rectangular elements based on paper substrate material present bandwidth of 340 and $290 \mathrm{MHz}$ respectively. Thus proposed paper based substrate material does offers a broadband frequency behaviour. 


\section{References}

[1] J. A. Encinar et al., "Dual-Polarization Reflectarray in Ku-band Based on Two Layers of Dipole-Arrays for a Transmit-Receive Satellite Antenna with South American Coverage," 2017 11th Eur. Conf. Antennas Propag., pp. 80-83, Mar. 2017.

[2] X.-C. Duan, Y.-X. Xia, Y. Zhou, B. Li, Y. Liu, and X. Lv, “A novel double-layered dielectric reflectarray,” in 2016 IEEE 9th UK-Europe-China Workshop on Millimetre Waves and Terahertz Technologies (UCMMT), 2016, pp. 94-95.

[3] A. Sarshar, "Design of a Stacked Stub-Loaded Patch Element for X-band Reflectarray Antenna with True Time Delay," pp. 3343-3346, 2013.

[4] B. D. Nguyen, K. T. Pham, V. S. Tran, L. Mai, and N. Yonemoto, "Reflectarray element using cut-ring patch coupled to delay line,” IEEE Antennas Wirel. Propag. Lett., vol. 14, pp. 571-574, 2015.

[5] J. A. Ortiz-Fuentes, J. Silva-Montero, J. I. Martinez-Lopez, J. Rodriguez-Cuevas, and A. E. Martynyuk, "Dualfrequency reflectarray based on split-ring slots," IEEE Antennas Wirel. Propag. Lett., vol. 16, pp. 952-955, 2017.

[6] J. H. Yoon, Y. J. Yoon, W. Lee, and J. So, "Broadband microstrip reflectarray with five parallel dipole elements," IEEE Antennas Wirel. Propag. Lett., vol. 14, pp. 1109-1112, 2015.

[7] R. S. Malfajani and Z. Atlasbaf, "Design and implementation of a dual-band single layer reflectarray in X and K bands," IEEE Trans. Antennas Propag., vol. 62, no. 8, pp. 4425-4431, 2014.

[8] J. Stockmann and R. Hodges, "The use of waveguide simulators to measure the resonant frequency of ku-band microstrip arrays," in IEEE Antennas and Propagation Society, AP-S International Symposium (Digest), 2005, vol. 1, pp. 417-420.

[9] H. Rajagopalan and Y. Rahmat-Samii, "Dielectric and Conductor Loss Quantification for Microstrip Reflectarray: Simulations and Measurements," IEEE Trans. Antennas Propag., vol. 56, no. 4, pp. 1192-1196, Apr. 2008.

[10] B. S. Cook and A. Shamim, "Utilizing Wideband AMC Structures for High-Gain Inkjet-Printed Antennas on Lossy Paper Substrate," IEEE Antennas Wirel. Propag. Lett., vol. 12, pp. 76-79, 2013.

[11] M. Kanagasabai and J. Kizhekke Pakkathillam, "Performance evaluation of a dual band paper substrate wireless sensor networks antenna over curvilinear surfaces," IET Microwaves, Antennas Propag., Jan. 2015. 